\title{
Rhythmic Motion Analysis using Motion Capture and Musical Information
}

\author{
Takaaki Shiratori \\ Dept. of Information and \\ Communication Engineering \\ The University of Tokyo \\ Tokyo, 153-8505, Japan \\ siratori@cvl.iis.u-tokyo.ac.jp
}

\author{
Atsushi Nakazawa \\ Cybermedia Center \\ Osaka University \\ Osaka, 560-0043, Japan \\ nakazawa@ime.cmc.osaka-u.ac.jp
}

\author{
Katsushi Ikeuchi \\ Institute of Industrial Science \\ The University of Tokyo \\ Tokyo, 153-8505, Japan \\ ki@cvl.iis.u-tokyo.ac.jp
}

\begin{abstract}
The number of Japanese traditional dancers has been decreasing. Without performers, some dances will be disappeared, because they can not be recorded by conventional media, such as paper. We have proposed an archiving method specifically for dancing pattern. Our method has four main stages: 1. Digitizing motions by motion capture systems, 2. Analyzing motions, 3. Synthesizing motions, 4. Reproducing the dance motions by $C G$ and humanoid robots. In order to effectively record the moving patterns, motion primitives are extracted. Each motion primitive describes each basic motion. However, most previous primitive extraction fails to provide the rhythm which results in unrhythmic synthesized motion. In this paper, we propose a new motion analysis method, which integrates music rhythm into the motion primitives. Our experiment confirmed that our motion analysis yielded the motion primitives in accordance to the music rhythm.
\end{abstract}

\section{Introduction}

Japanese traditional dances are personally taught from one person to another person. Due to the decreasing number of dancers, many of them will be soon disappeared. In order to conserve the traditional dances, we have developed a digital archiving method specifically for dancing pattern as shown in Figure 1.

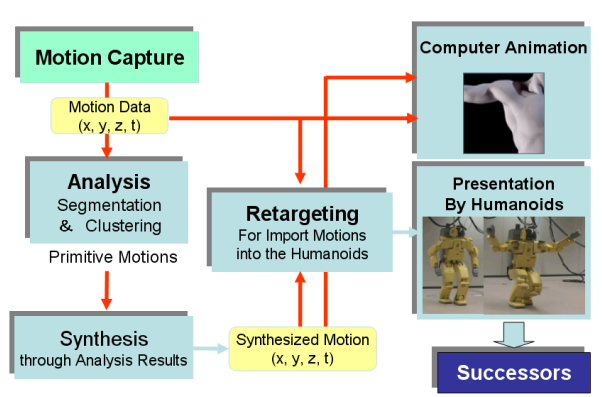

Figure 1. Project Overview
A motion capture system is one of the most effective methods for digitizing human motions, and commonly used to generate human motions in computer graphics. The system provides only the low-level information(motion). Increasing the the number of data is used to cope with the lack of high-level information(E.g. moving rhythm). Further information is interpolated from the data. Interpolation can be done by "motion graph"[1][5][6], filter banks[2] etc.

Dances can be considered as the special kind of motion and required the special kind of motion analysis. Motion beats[4] was proposed to capture the rhythmic motion. Interfaced system proposed in [8] made it easy to edit and synthesize the dance motions.

Japanese traditional dances cannot be effectively analyzed by conventional methods, because every dance moiton is represented as a sequence of "primitive motions", whose spans are based on a rhythm and correspond to the rhythmic pattern underlying the motion. Motion primitive is defined as the basic motion. A complex movement can be described by the sequence of motion primitives. In Japanese traditional dances, primitive motions are called Mai.

In our previously proposed method[7], motion primitives are extracted based on the speed of hands and feet. However, the results sometimes lose the essence of a dance and the synthesized motion is not always in tune with the music, because some of Mai, the primitive motions, are segmented. In this paper, music rhythm is included in our motion-analysis method to create the motion in tune with the music.

An overall of our proposed method is presented in Section 2. We detect the music rhythm by beat tracking method([3]) in Section 3. Our segmentation method for extraction of motion primitives is described in Section 4. Our method was then verified and compared with another method in Section 5. The paper is concluded in Section 6.

\section{Algorithm Overview}

An overall of our proposed method is shown in Figure 2. First, speed sequence of hands and center of mass is caluculated and candidates for segmentation are extracted from speed sequence. Then, if there is the candidate around the music rhythm, each speed sequence of hands and center of 
mass is segmented at the music rhythm. Finally, motion sequence is segmented by logical operation of each result of hands and center of mass.

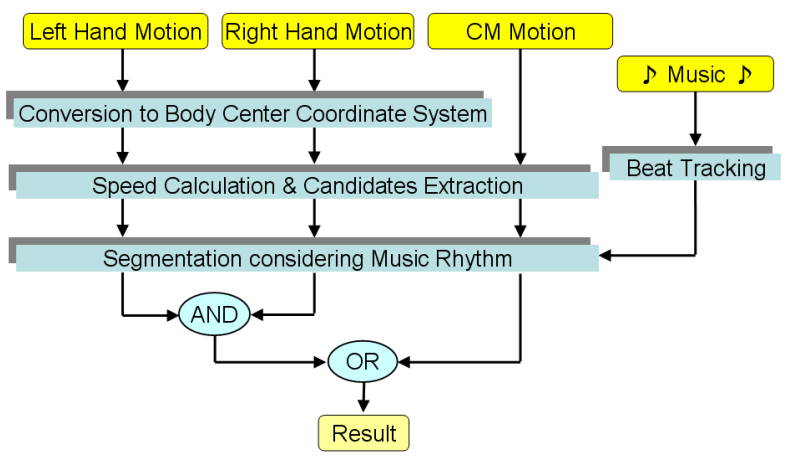

Figure 2. Algorithm Overview

\section{Beat Tracking Method}

We adopted the beat tracking method proposed in [3], which is used for detecting rhythm in western music.

\subsection{Musical Elements for Beat Tracking}

There are three kinds of musical elements for beat tracking:

- Onset times

- Chord Changes

- Percussion Patterns

Experiments in [3] showed that the three musical elements were suitable for western music, such as pop, rock, etc. However, Japanese dance music is different to western music in both chord and rhythm. In this section, we first evaluate and select musical elements appropriated to Japanese music. Then we describe the extracting formulation and the processing of the desired musical element.

\subsubsection{Onset times}

One of the good candidates for beat tracking agent is onset times(rise time). In Japanese dance music, there are few rhythm changes, such as rit., accel., etc. Therefore, onset times is very effective for beat tracking.

\subsubsection{Chord Changes}

The frequency characteristic of chord in western music is different from that in eastern music. In addition, Japanese dance music hardly changes chord. Therefore, this musical element is not efficient for tracking beat in Japanese music.

\subsubsection{Percussion Patterns}

The percussion pattern is extracted based on the spectrum pattern of percussion instruments(E.g. drum, etc.). However, percussion instruments are not necessary in Japanese dance music. Furthermore, [3] recognized percussion patterns from bass drums and snare drums. Both are not used in Japanese dance music. Hence, this musical element is not important in Japanese dance music.

From the above evaluation, we conclude that the rhythm in Japanese dance music can be effectively extracted by onset times. The other two musical elements are unnecessary.

\subsection{Onset Component Extraction}

When a sound is produced, the spectral power corresponding to the frequency of that sound will increase. Each onset component is extracted as shown in Figure 3.

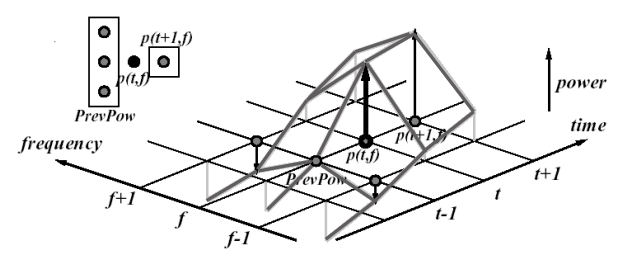

Figure 3. Onset Component Extraction: Calculating how much power increases from "PrevPow"

The magnitude of increasing spectral power $d(t, f)$ at time $t$ for frequency $f$ is defined as:

$$
d(t, f)=\left\{\begin{aligned}
& \max (p(t, f), p(t+1, f))-\text { PrevPow }(\min (p(t, f), p(t+1, f)) \geq \text { PrevPow }) \\
& 0 \quad(\text { otherwise }),
\end{aligned}\right.
$$

where

$$
\text { PrevPow }=\max (p(t, f-1), p(t-1, f), p(t-1, f+1)),
$$

and $p(t, f)$ is the spectral power at time $t$ and frequency $f$.

PrevPow considers the spectral power between $f-1, f$ and $f+1$ at time $t-1$. Therefore, an error onset time is not picked up when the musical frequency is deviated by noise, players' skill, etc.

Next, at the frequency bands a user selected, beat tracking system calculates $D(t)$ defined as follows:

$$
D(t)=\sum_{\text {ChosenFreqBands }} d(t, f),
$$

Frequency range is divided into 7 bands $(0-125 \mathrm{~Hz}, 125-$ $250 \mathrm{~Hz}, 250-500 \mathrm{~Hz}, 500-1000 \mathrm{~Hz}, 1 \mathrm{k}-2 \mathrm{kHz}, 2 \mathrm{k}-4 \mathrm{kHz}$ and more than $4 \mathrm{kHz}$ ). Each band corresponds to one octave as sensed by human. 


\subsection{Estimation of Beat Start and Beat Interval}

In this section, we describe how we estimate beat start and beat interval.

First, auto-correlation function $(\Phi(\tau))$ of $D(t)$ is calculated. Auto-correlation function indicates the orthogonality of $D(t)$ and $D(t+\tau)$ and is defined as follows:

$$
\Phi(\tau)=\frac{1}{N} \sum_{t=1}^{N} D(t) D(t+\tau)
$$

where $\mathrm{N}$ is the number of the data.

In $\Phi(\tau)$, if a peak appear at $\tau_{\max }, D(t)$ is very similar to $D\left(t+\tau_{\max }\right)$. And, $\tau_{\max }$ is a beat interval(Figure 4$)$.

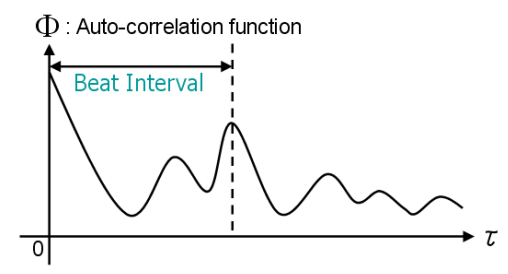

Figure 4. Beat-interval Estimation

Beat start time $\left(t_{\mathrm{st}}\right)$ is calculated from beat interval $\tau_{\max }$ as follows:

$$
\begin{gathered}
t_{\mathrm{st}}=\arg \max _{t_{\mathrm{st}}} \sum_{i=0}^{M} D\left(t_{\mathrm{i}}\right), \\
\text { where } t_{\mathrm{i}}=t_{\mathrm{st}}+i * \tau_{\max } \quad(0 \leq i \leq M) .
\end{gathered}
$$

\section{Motion Analysis Considering Music Rhythm}

We hypothesize that the sequence of human motion consists of motion elements which we define as "primitive motions." The primitive motions are the set of basic motions and this concept has a great advantage for human motions processing(archive, edit, etc).

Japanese traditional dance is the sequence of short dance("Mai"). This short dance is used as the primitive motion in Japanese traditional dance. In between the short dance, dancers stop moving. So it is possible to extract the primitive motion(short dance) based on the stop point. The stop point often appears at music rhythm which can be detected by onset components, because the spans of the primitive motions are based on the music rhythm and correspond to the rhythmic pattern underlying the motion.

The overall analysis method for human dance motions is presented in this section.

\subsection{Motion Elements for Analysis}

Our analysis method is based on the speed of a performer's hands and center of mass(CM). In most dances including Japanese traditional dance, movement of hands is related with the whole body expression. Therefore, the speed of the hands is useful for extracting the stop motions; however, it is not sufficient for primitive extraction as presented in the following two cases.

\section{- Case : The dancer loses sense of rhythm}

- When a dancer missteps(according to the music rhythm), he tends to correct his dance by lengthening/shortening his steps. Such movements make the detection of the stop motions loose, since the speed of body parts are not low enough to be detected as stop motion. Consequently, a stop motion is not effectively extracted by only the speed of the hands.

- Case : Dances are varied by the preference and the gender of performers.

- Japanese traditional dances are taught from one person to another persons without the proper record. Consequently, even in the same dances, different dancers performs differently. While the difference among professional dancers is not visually distinct, it has much effects on motion analysis.

In addition to the motion of the hands, our algorithm use the motion of the body's CM. The motion of CM represents the motion of the whole body; thus, the effects of misstep and individual difference are less. Primitives can then be effectively extracted. The calculation of the body's CM is derived from Figure 5:

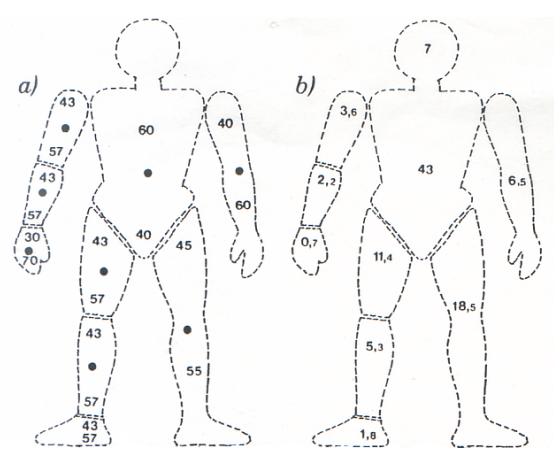

Figure 5. Center of Mass

Figure(a) presents where the position of the center of mass of each body part is and Figure (b) presents the percentage of mass of each body part.

\subsection{Segmentation of Dance Motions considering Music Rhythm}

There are three steps to segment the motion sequence.

1. Speed Calculation

2. Segmentation Candidates Extraction

3. Segmentation Considering Music Rhythm 


\subsubsection{Speed Calculation}

The captured motion data is recorded in global coordinate system. To calculate speed of the hands, we define the local coordinate system as follows. The origin of the local coordinate system is the middle of human waist. The three axis of the local coordinate are shown in Figure 6. $x$ axis is parallel to the waist, $y$ axis is perpendicular to the waist and $z$ axis is vertical. Relative motion of individual body part to the entire body is, therefore, the motion in local coordinate system.

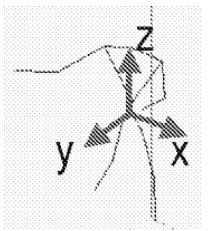

Figure 6. Body Center Coordinate System

On the other hand, the CM is calculated in the global coordinate system.

The effect of noise is reduced by smoothing motion sequence with gaussian filter before extracting candidates.

\subsubsection{Segmentation Candidates Extraction}

After calculating speed, the systems extracts the candidates for segmentation. The candidates are defined as the localminimum point which satisfies the following two conditions(Figure 7):

1. Each candidate is a local minimum in speed sequence and the local minimum is less than the minimum-speed threshold.

2. The local maximum between two successive candidates is larger than the maximum-speed threshold.

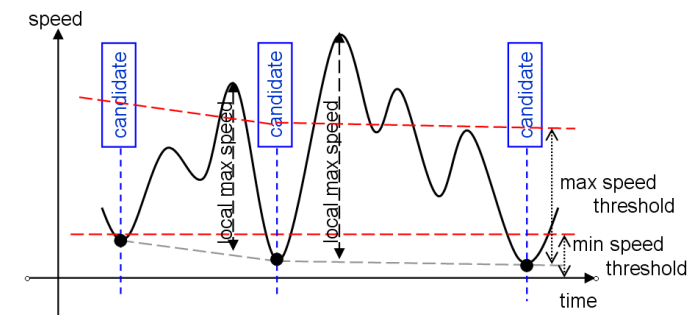

Figure 7. Segmentation Candidates Extraction

\subsubsection{Segmentation Considering Music Rhythm}

At each speed sequence, our method tests whether there are candidates around music rhythm detected by onset times $\left(t_{\text {beat }}\right)$. If there is a candidate, it is possible that there is a stop point around $t_{\text {beat }}$ and the motion sequence is segmented at $t_{\text {beat }}$. Figure 8 illustrates the segmentation process.
In the figure, there are no segmentation candidates around 1 st and 3rd music rhythm and the motion is not segmented at these points. Because there are segmentation candidates around 2 nd and 4 th music rhythm, the motions are segmented at these points.

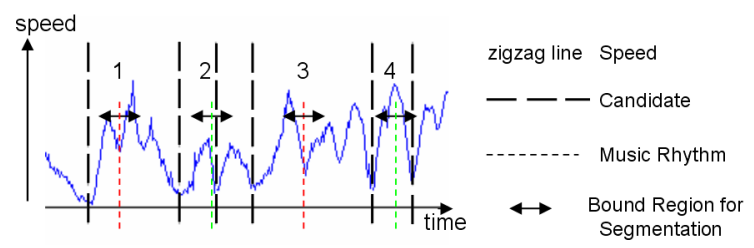

\section{Figure 8. Segmentation considering Music Rhythm}

Logical operation is used to confirm whether $t_{\text {beat }}$ is the stop point. The operation checks the harmony of both hands and the $\mathrm{CM}$ and is defined as:

\section{(Result of Left Hand $\cap$ Result of Right Hand) $\cup$ Result of Center of Mass.}

AND operation is applied because the dancer stop moving entirely during the stop between primitives. OR operation is applied, so that the stop point undetected by hand motion is compensately detected from the motion of CM.

\section{Results}

\subsection{Experimental Data}

Our proposed method was evaluated using three dance segments: male and female Aizu-bandaisan, and Jongara-bushi. These motion data were captured by Vicon Motion Systems, which is optical motion capturing system and records the position of 33 markers on a person. The sampling rate of Aizubandaisan dance and Jongara-bushi were $120 \mathrm{fps}$ and 200fps, respectively.

The music was stored in the wav format. The data size was 16 bits and the sampling rate was set at $32000 \mathrm{~Hz}$.

\subsection{Results of Beat Tracking}

To extract the onset components, frequency spectrum was calculated by FFT. The number of samples for FFT was 1024 samples. The window function for FFT was gaussian function which is suitable for audio signal processing and was shifted by 256 samples.

The estimated beat interval of Aizu-bandaisan and Jongara-bushi dance music were 0.704 second(about $d=85$ ). and 0.576 second(about $d=104$ ), respectively. In Figure 9, the upper left window was the spectrogram and vertical lines were the estimated rhythm. The lower left window was $D(t)$ of each frequency band described. The rhythm appeared at the onset times which was the deep gray in the spectrogram. 


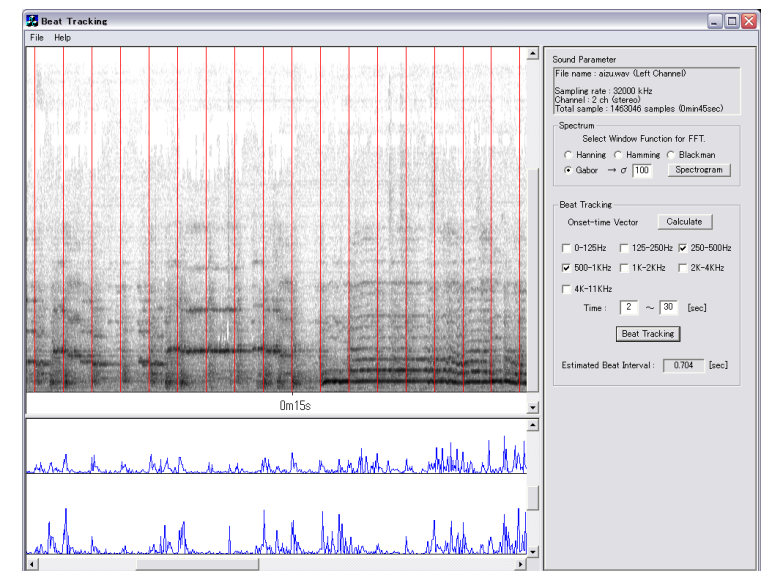

Figure 9. Result - Beat Tracking of Aizubandaisan

\subsection{Results of Motion Segmentation considering Music Rhythm}

In all dances, we compared the result from our segmentation by the motion of the $\mathrm{CM}$ and the hands with the one from other method.

\subsubsection{Female Aizu-bandaisan dancer}

The result of our method is shown in Figure 10. There was only one error in the result(9th frame). On the other hand, the segmentation based on the hand motions oversegmented the motion. Out of the 15 detected stop point, 6 were errors. In addition, the method failed to extract one stop point.

\subsubsection{Male Aizu-bandaisan Dancer}

In this dance, a male dancer mis-steped many times. During the mis-step, he retuned his motion to the music, and could not completely stop his motion. Our proposed method could successfuly extracted most of the stop point, and only two were missed(Figure 11). On the other hand, the segmentation based on the hands motion and music without CM motions gave a few number of false stop points as well as misdetected stop points. These results implied that the error caused by individual difference can be reduced by considering the $\mathrm{CM}$ motions.

From our proposed segmentation, the result of female dancer was compared to that of male dancer as shown in Figure 12 . Both of the results were very similar. The similarity implied that the proposed method overcame the difference in motion sequence derived from the variations of dance.

\subsubsection{Jongara-bushi}

The result of our method is shown in Figure 13. There were 1 misdetected error and 1 undetected error in the result. On the other hand, the segmentation based on the hand motions failed to extract some stop points. Out of the 6 detected stop point, there were no errors, but the method failed to extract 6 stop points.

Our segmentation by motion from the $\mathrm{CM}$ and the hands gave superior results than the one by the motion of the hands only. It indicated that our segmentation could be effectively used for extracting motion primitives.

\section{Conclusion}

This paper described a new analysis method for the motion of human dance. Our method considers music rhythm and the motion sequence of the center of mass as well as the hands.

The previous methods consider the speed of hands and feet or joint roration and result in that the motion sequence is divided into too many segments. So the previous method is not efficient for archiving. Proposed method considering music rhythm makes it possible to divide the dance sequence into the approriate segments called Mai, and most of Mai are not oversegmented. In addition, these segments are considered music rhythm and the synthesized motions consisting of primitive motions will be rhythmic in CG.

Our proposed method also considers the speed of center of mass to understand the movement of the body. The analysis methods without considering the center of mass cannot deal with the case that there are some variations derived from misstep or gender difference etc., while our proposed method can solve these problems.

We plan to include the analysis of feet motions in our future system. Furthermore, we also plan to add other information besides motion into our primitives. The information will be selected based on the further discussion with professional dancers.

\section{References}

[1] O. Arikan and D. A. Forsyth. Interactive motion generation from eamples. In Proc. of ACM SIGGRAPHO2, pages 483-490, 2002.

[2] A. Bruderlin and L. Williams. Motion signal processing. In Proc. of ACM SIGGRAPH95, pages 97-104, 1995.

[3] M. Goto. An audio-based real-time beat tracking system for music with or without drum-sounds. Journal of New Music Research, 30(2):159-171, June 2001.

[4] T. Kim, S. I. Park, and S. Y. Shin. Rhythmic-motion synthesis based on motion-beat analysis. In Proc. of ACM SIGGRAPH95, 2003.

[5] L. Kovar and M. Gleicher. Motion graph. In Proc. of ACM SIGGRAPH02, pages 473-482, 2002.

[6] J. Lee, J. Chai, P. S. A. Reitsma, J. K. Hodgins, and N. S. Pollard. Interactive control of avatars animated with human motion data. In Proc. of ACM SIGGRAPHO2, 2002.

[7] A. Nakazawa, S. Nakaoka, K. Ikeuchi, and K. Yokoi. Imitating human dance motions through motion structure analysis. In Proc. of International Conference on Intelligent Robots and Systems, pages 2539-2544, October 2002.

[8] Y. Yoshida, Y. Matsuoka, and K. Hachimura. Processing of human body movement based on lavanotation labanreader and labaneditor. IPSJ, 1998. 


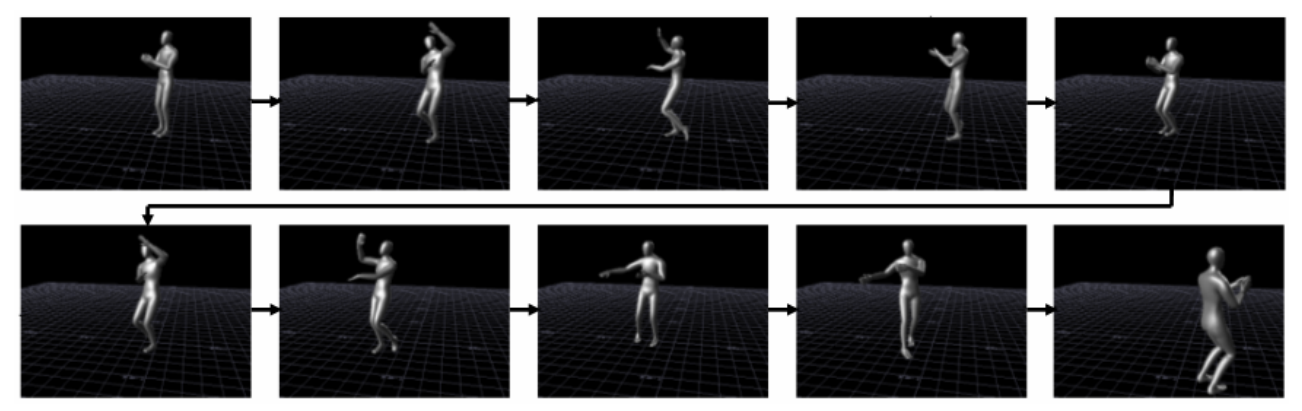

Figure 10. Segmentation result - Aizu-bandaisan dance(female)
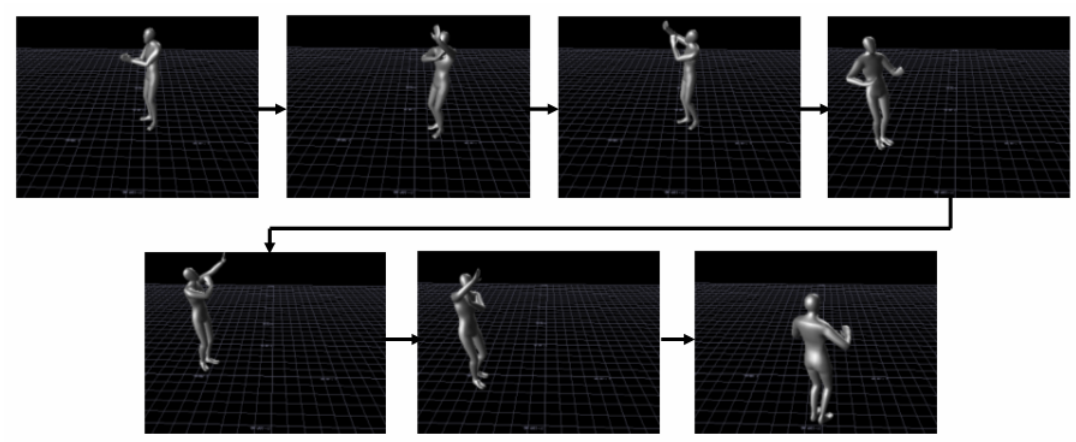

Figure 11. Segmentation result - Aizu-bandaisan dance(male)
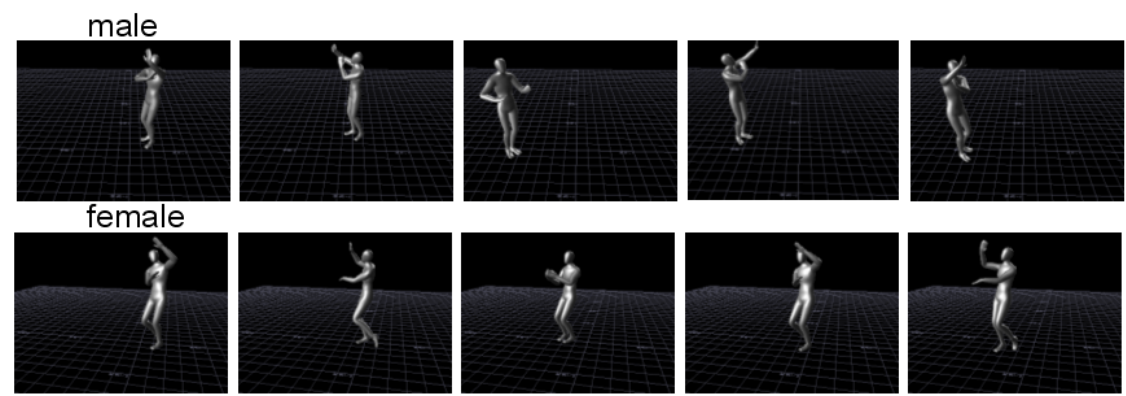

$\stackrel{\mathrm{time}}{\longrightarrow}$

Figure 12. Comparing male segments with female ones

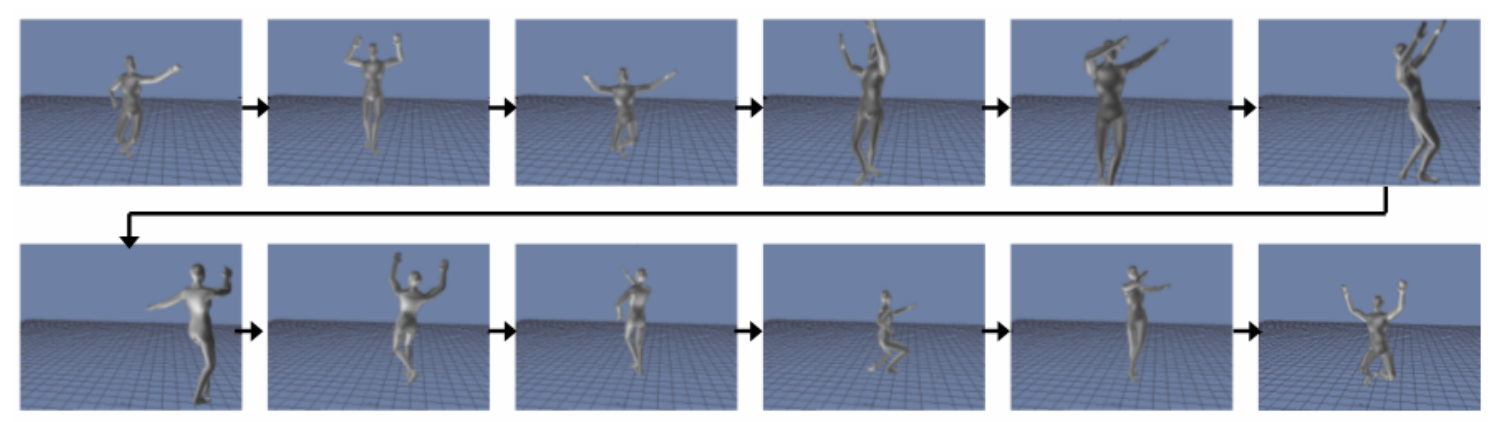

Figure 13. Segmentation result - Jongara-bushi 